\title{
Online Symptom Checker Applications: Syndromic Surveillance for International Health
}

\author{
Andrew C. Berry, DO \\ Division of Gastroenterology, Larkin Community Hospital, South Miami, FL
}

Patient demand continues to outpace healthcare access globally, particularly in resource-limited regions, so finding nontraditional strategies to mitigate this healthcare challenge is imperative. ${ }^{1}$ In addition, healthcare facilities can become dangerous places for disease amplification and vectors for community outbreaks. ${ }^{2}$ Innovative modalities have the potential to provide a sterile avenue to mitigate such outbreaks, to address the diagnostic and triage demands of the community, and to potentially monitor epidemiologic trends.

Symptom checkers are online applications that allow users to input symptoms and obtain a diagnostic and triage decision. Symptom checkers provide a way to reach international populations in a cost-effective manner and to adapt to the societal demand for on-demand electronic healthcare, as approximately $75 \%$ of international web users search for health information online. ${ }^{3}$

Syndromic surveillance is the real-time (or near real-time) collection, analysis, interpretation, and dissemination of health-related data that enable early identification of the impact (or absence of impact) of human public health threats that require effective public health action. ${ }^{4}$ Syndromic surveillance relies on clinical signs, epidemiologic trends, and proxy measures (eg, absenteeism, drug sales, doctor visits) that create a provisional diagnosis, or syndrome. ${ }^{4}$ As part of the global infrastructure, syndromic surveillance can become a way to monitor disease, respond quickly, and allocate resources to minimize potential outbreaks. Syndromic surveillance has evolved since the early 2000s, with successful tests and opportunities for improvement occurring as a result of worldwide natural disasters (flooding, fires, volcanic eruptions), pandemic outbreaks (2009 influenza), and mass gatherings and sporting events (Olympic Games). ${ }^{4-6}$

Prior to 2012, real-time monitoring only consisted of calls to telephone health advice lines in combination with a partial snapshot of general practitioner (GP) surveillance network monitoring. ${ }^{5}$ The 2012 Winter Olympic Games offered new syndromic surveillance modalities, including GP out of hours and daily emergency department symptom and diagnostic uploads. ${ }^{5}$ After the 2012 Olympics, online symptom checkers were trialed as a potential addition to syndromic surveillance with great success and popularity. ${ }^{7} \mathrm{~A}$ strong correlation existed between the online symptom checker and traditional telephone triage data for a number of syndromic indicators. ${ }^{7}$ For some disease systems (eg, respiratory), online symptom checker data appeared to provide additional early warning over telephone triage health data. ${ }^{7}$ These promising early findings suggest that symptom checkers combined with real-time data collection can be used to monitor local and worldwide deviations from epidemiologic patterns, an outcome even more valuable than the initially envisioned diagnostic and triage potential.

An accurate and reliable symptom checker is not only important for consumer diagnostic and triage capabilities and for monitoring alarms or deviations from epidemiologic norms, but it is imperative for formulating an accurate epidemiologic baseline. A large-scale audit of online symptom checkers identified many inconsistences between symptom checkers in regard to diagnostic and triage accuracies, with further variations associated with disease type and severity. ${ }^{8}$ Further analysis retrospectively and indirectly pitted the doctor vs the symptom checker, suggesting that the doctor outperforms the symptom checker. ${ }^{9}$ Prospective, head-to-head comparison studies have revealed some diagnostic and triage inconsistencies with symptom checker use and real-life, complex patient data, spurring efforts to refine baseline algorithmic accuracies. ${ }^{10,11}$

Initial critiques called for consecutive, prospective, realpatient cases with well-validated diagnoses. The Human Diagnosis Project is a worldwide effort, created and led by the global medical community, that is spearheading an open intelligence system to map the steps to help any patient or provider. ${ }^{12}$ This system mimics the success of Wikipedia and other open intelligence systems. Physicians can contribute real patient cases, and their physician peers can analyze, solve, or expand on the cases. ${ }^{12}$ The goal is to build a vast algorithmic array of real patient data so that doctors can better address the complex, dynamic, real-life patient-not forgetting the potential for an accurate baseline for syndromic surveillance. Combining the intelligence of doctors with machine learning has the potential to provide accurate, affordable, and accessible care internationally. ${ }^{1}$

The true value of online symptom checkers resides not in their direct ability to help diagnose and triage the ondemand healthcare-seeking patient but in the indirect gains obtained from their use in international public health surveillance. Use of symptom checkers can provide real-time data monitoring of patient symptoms, forewarning healthcare officials in resource-limited communities. Officials can thus allocate resources accordingly and stay ahead of many illnesses and disease outbreaks. The symptom checker is a valuable tool that healthcare personnel must delve into further to 
harness its true potential-international health syndromic surveillance.

\section{REFERENCES}

1. Morita T, Rahman A, Hasegawa T, Ozaki A, Tanimoto T. The potential possibility of symptom checker. Int $J$ Health Policy Manag. 2017 Apr 5;6(10):615-616. doi: 10.15171/ijhpm.2017.41.

2. Allegranzi B, Kilpatrick C, Storr J, Kelley E, Park BJ, Donaldson L; Global Infection Prevention and Control Network. Global infection prevention and control priorities 2018-22: a call for action. Lancet Glob Health. 2017 Dec;5(12):e1178-e1180. doi: 10.1016/S2214-109X(17)30427-8.

3. Doherty-Torstrick ER, Walton KE, Fallon BA. Cyberchondria: parsing health anxiety from online behavior. Psychosomatics. 2016 Jul-Aug;57(4):390-400. doi: 10.1016/j.psym.2016.02.002.

4. Triple $S$ Project. Assessment of syndromic surveillance in Europe. Lancet. 2011 Nov 26;378(9806):1833-1834. doi: 10.1016/S0140-6736(11)60834-9.

5. Elliot AJ, Morbey RA, Hughes HE, et al. Syndromic surveillance a public health legacy of the London 2012 Olympic and Paralympic Games. Public Health. 2013 Aug;127(8):777-781. doi: 10.1016/j.puhe.2013.05.007.

6. Todkill D, Hughes HE, Elliot AJ, et al. An observational study using English syndromic surveillance data collected during the 2012 London Olympics - what did syndromic surveillance show and what can we learn for future mass-gathering events? Prehosp Disaster Med. 2016 Dec;31(6):628-634.
7. Elliot AJ, Kara EO, Loveridge $P$, et al. Internet-based remote health self-checker symptom data as an adjuvant to a national syndromic surveillance system. Epidemiol Infect. 2015 Dec;143 (16):3416-3422. doi: 10.1017/S0950268815000503.

8. Semigran $\mathrm{HL}$, Linder JA, Gidengil C, Mehrotra A. Evaluation of symptom checkers for self diagnosis and triage: audit study. BMJ. 2015 Jul 8;351:h3480. doi: 10.1136/bmj.h3480.

9. Semigran HL, Levine DM, Nundy S, Mehrotra A. Comparison of physician and computer diagnostic accuracy. JAMA Intern Med. 2016 Dec 1;176(12):1860-1861. doi: 10.1001/jamainternmed .2016 .6001 .

10. Berry AC, Cash BD, Mulekar MS, Wang B, Melvin A, Berry BB. Symptom checkers vs. doctors, the ultimate test: a prospective study of patients presenting with abdominal pain. Gastroenterology. 2017 Apr;152(5):S852-S853. doi: 10.1016/ S0016-5085(17)32937-2.

11. Berry AC, Berry NA, Wang B, Mulekar M, Melvin A, Berry BB. Symptom checkers vs. doctors: a prospective, head-to-head comparison for GERD vs. non-GERD cough. Am J Gastroenterol. 2017 Oct;112(S):S190.

12. The Human Dx. Human diagnosis project. https://www .humandx.org. Published 2018. Accessed April 15, 2018. 